\title{
Adiposcience and adipotoxicity
}

\author{
Kazuwa Nakao
}

Traditionally, adipose tissue was viewed as a rather inert organ that functioned solely as a fat storage depot. However, this tissue is now recognized as a bona fide endocrine organ that has the ability to secret numerous adipokines, including leptin.

Excess storage of fat in adipose tissue (i.e. obesity) is accompanied by ectopic lipid deposition in nonadipose tissues, such as the liver, skeletal muscles and pancreas. The negative effects of ectopic lipid deposition on glucose metabolism have been proposed to reflect a state of 'lipotoxicity', in which leptin is implicated as an antilipotoxic hormone. Over the past decade, evidence has accumulated that the adipose tissue has multiple functions in both normal physiology and disease. These functions are affected by changes in the adipose-tissue mass and/or the distribution of fat in subcutaneous and visceral adipose tissues. These changes result in dynamic alteration of adipokine production that reflect the severity of obesity, chronic inflammation with the infiltration of macrophages, leptin resistance, and altered autonomic nervous function, in addition to insulin resistance and lipotoxicity. I, therefore, propose the term 'adipotoxicity' to describe the negative effects associated with obesity. Adipotoxicity can be defined as the sum of the negative effects associated with storage of excess fat in adipose tissue on obesity-related clinical features, such as diabetes mellitus and arteriosclerosis. As a consequence, the metabolic syndrome-an obesity-related cluster of diabetes mellitus, dyslipidemia and hypertension-should be studied from a comprehensive viewpoint that is based on the concept of adipotoxicity.

Detailed comparisons of obesity and generalized lipodystrophy (i.e. lack of adipose tissue) have contributed substantially to our understanding of adipotoxicity. In particular, ....further

studies on

adipotoxicity

will hopefully

provide a new

strategy that

we can exploit

to prevent and

treat obesity...

KNakao is the

Chairman of

the Department of

Medicine and Clinical

Science at Kyoto

University Graduate

School of Medicine,

Kyoto, Japan.

\section{Competing interests}

The author declared no competing interests.

www.nature.com/clinicalpractice doi:10.1038/ncpendmet1052 the dramatic effects of leptin-replacement therapy on patients with lipodystrophy disclosed subtle functions of the adipose tissue, and became the paradigm used to elucidate the mechanisms of adipotoxicity. We should, however, remember that our current understanding of the function of adipose tissue is far from complete. Further studies on adipotoxicity will hopefully provide a new strategy that we can exploit to prevent and treat obesity and the metabolic syndrome.

Interpretation of the results of different studies that focus on obesity can be hampered by use of the misleading prefixes 'lipo' and 'adipo'. For example, lipogenesis is used to describe the metabolic formation of lipid, whereas adipogenesis refers to the differentiation of preadipocytes into mature adipocytes, the main function of which is to store fat. Of course, lipogenesis and adipogenesis are closely linked biological processes. Furthermore, a key aim of the study of obesity is to understand the significance of the storage of excess fat in the adipose tissue. I feel it is appropriate, then, to coin the term 'adiposcience' to describe studies that aim to evaluate the relationship between adipogenesis and obesity. The definition of adiposcience might also be extended to cover other aspects of metabolism, from appetite regulation to energy expenditure, as well as their mechanisms of action on the adipose tissue.

The prevalence of obesity and its comorbidities has now reached pandemic proportions. Indeed, the main theme of the 13th International Congress of Endocrinology (held during 8-12 November 2008 in Rio de Janeiro) was obesity and the metabolic syndrome. When the Congress reconvenes in Kyoto in March 2010, however, the concepts of adiposcience and adipotoxicity clearly should be at the forefront of our discussions. 